\title{
Scapuloperoneal syndrome with cardiomyopathy: report of a family with autosomal dominant inheritance and unusual features
}

\author{
A CHAKRABARTI, JMS PEARCE \\ From the Department of Neurology, Hull Royal Infirmary, Hull, UK
}

SUMMARY Four members of a family with scapuloperoneal syndrome were examined and investigated. The pattern of inheritance was autosomal dominant and the myopathic basis of the muscle atrophy was established by histological studies of muscle and spinal cord. The family illustrates an unusual combination of features which appear to be distinct from those of other families with myopathic scapuloperoneal syndrome and autosomal dominant inheritance. These include early age of onset and rapid progression in most cases; occurrence of early muscle contractures; and a high incidence of severe cardiomyopathy in three of the four cases. Some of these features resemble those seen in the $x$-linked form of the disease and the present family appeared to be a new variant of the autosomal dominant form of the scapuloperoneal syndrome.

A century after its first recognition by Brossard ${ }^{1}$ in 1886 , the nosology of the syndrome of genetically determined scapuloperoneal atrophy remains complex. The term "scapuloperoneal amyotrophy" was coined by Davidenkow ${ }^{2}$ who described the characteristic features of the syndrome. The age of onset was around 20 years, but in some cases as late as 45 . Inheritance was autosomal dominant and muscular weakness and wasting predominantly involved the shoulder girdle and upper arm muscles and anterior tibials and peronei. Progression of weakness was slow and sensory disturbances were seldom absent. Davidenkow ${ }^{2}$ however, reported no autopsy studies and could not establish whether the syndrome constituted a separate entity or was simply a variant of peroneal muscular atrophy. Over the last two decades several families with muscular atrophy in scapuloperoneal distribution have been described with muscle histology and EMG studies. The spectrum observed in the families differs in several respects: age of onset, rate of progression, pattern of transmission, pattern of muscular atrophy (neurogenic or myogenic) and the presence or absence of sensory changes. It appears therefore that the term scapuloperoneal syndrome has been applied to a hetero-

Address for reprint requests: Dr JMS Pearce, Hull Royal Infirmary, Hull HU3 2JZ, UK.

Received 28 May 1981 and in revised form 6 August 1981 Accepted 1 September 1981 genous group of neurogenic and myogenic disorders $\frac{1}{\overparen{D}}$ with different modes of inheritance and different $\frac{\circ}{8}$ 을 clinical courses. The nosological problem has been $\_$further complicated by the occasional discordance 0 between EMG and biopsy data reported in some 8 families $^{34}$ and evidence of both neurogenic and myogenic changes in the same patient..$^{5} \mathrm{~A}$ working classification of the syndrome, which has been $\rightleftharpoons$ proposed $^{4}$ is detailed in table 1 in a slightly modified

\section{Table 1 Scapuloperoneal syndrome-classification}

1 Scapuloperoneal muscular dystrophy (myopathic), autosomal dominant or sporadic, late onset (Ref 6-9).

2 Scapuloperoneal muscular atrophy (neurogenic)

(a) without sensory involvement

(i) autosomal dominant or sporadic, late onset (Ref 3,10 )

(ii) autosomal recessive or sporadic, early onset (Ref 4,11-13)

(b) with distal sensory disturbance

(i) autosomal dominent or sporadic, late onset (Ref 2,14$)$

(ii) autosomal recessive, early onset (Ref 15 )

3 X-linked scapuloperoneal syndrome (Ref 16-19).

form. This classification is not as clear-cut as it may appear, since the neurogenic form with sensory loss (Davidenkow's syndrome) may occur as the phenotypic manifestation in families in which other members display a distal distribution of involvement in all four limbs. ${ }^{27}$

We report here a family with scapuloperoneal syndrome which illustrates a combination of features not previously described, distinct from the types 146 
already mentioned in the classification (table 1). In this family, the propositus (case 1) and both hei siblings and father were affected, all of whom were examined and investigated. Figure 1 shows the family tree.
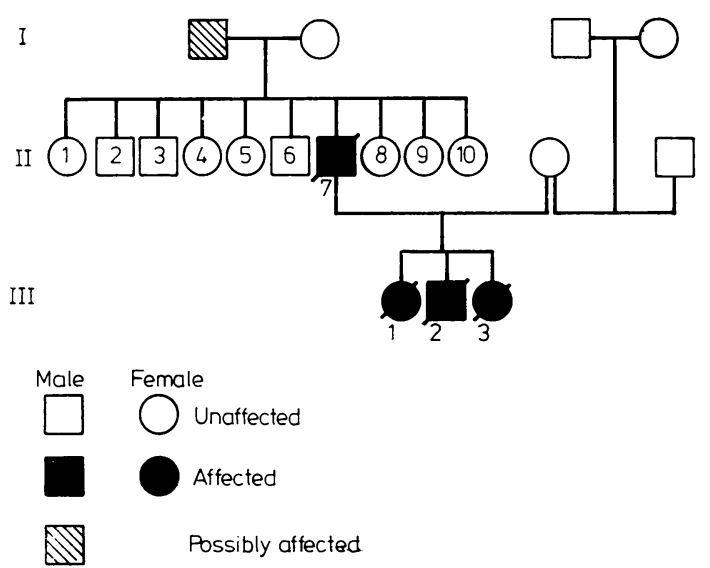

Fig 1 The family tree.

\section{Case histories}

Case 1 JD (111 1). A girl born in 1952, eldest of the three children of SD (11 117 ) was first seen at the age of 21 years. She was the product of a normal delivery at term and walked at one year. However an awkward gait was noticed at 18 months. Thereafter there was progressive weakness of the legs and later of the arms. From the age of 12 to $21 \mathrm{yr}$ there was little deterioration. On examination she had minimal weakness of sternomastoids, weakness and gross wasting of biceps and triceps with flexion deformities of both elbows with limitation of flexion to $70^{\circ}$. Weakness was slight in distal forearm muscles but marked in shoulder girdle muscles. She had marked wasting of anterior and posterior tibial muscles and knee extension was limited by flexion contracture to $150^{\circ}$. Proximal muscles of lower limbs were weak but not wasted. She had total tendon areflexia but no sensory abnormalities. Test for colour vision was normal. Serum CPK was 192 units/l (normal 0-50). At the age of $22 \mathrm{yr}$ she was admitted as an emergency with right hemiparesis and dysphasia. A similar episode had occurred two years earlier when the hemiparesis recovered in four and a half hours. Her neuromuscular disability remained unaltered. This time, the dysphasia cleared up in a few hours but the hemiparesis was slow to recover. She was in congestive cardiac failure and had tricuspid regurgitation. The cardiac silhouette was enlarged and ECG showed absence of atrial activity with a nodal escape rhythm (fig 2). She improved on diuretics. These findings suggested a cardiomyopathy with presumed endocardial thrombus formation and cerebral embolisation. Later, a left carotid angiogram was normal. She was readmitted at the age of $23 \mathrm{yr}$ with dense right hemiplegia and global dysphasia. Shortly

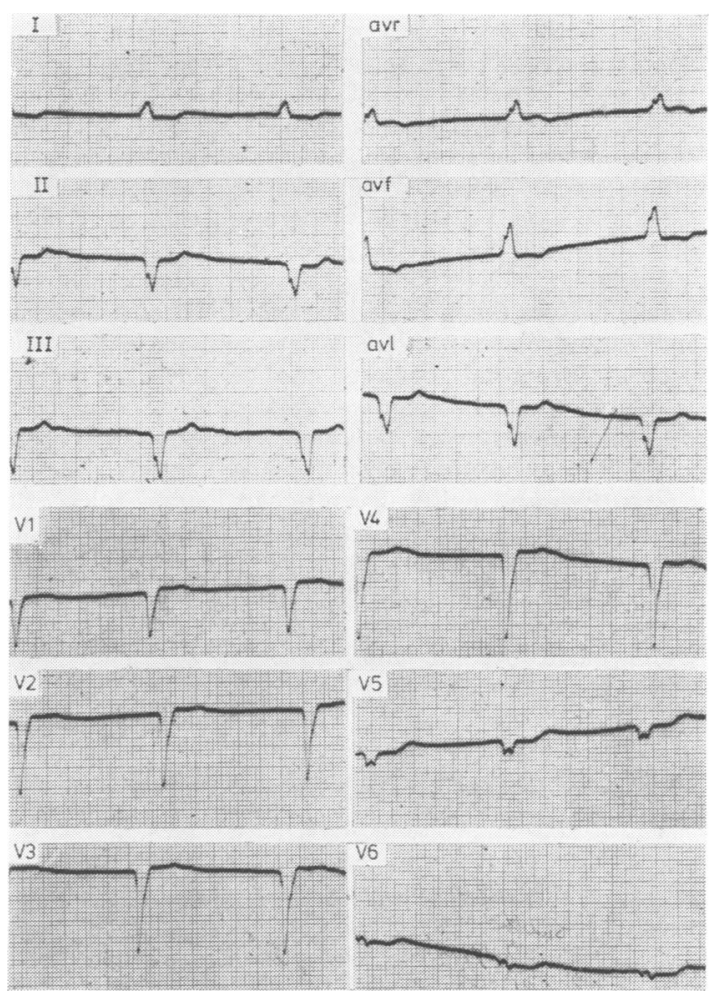

Fig 2 ECG in Case 1 showing left axis deviation, absence of $P$-waves and a nodal escape rhythm (45/ $\mathrm{min})$

after admission she lapsed into a coma and remained unconscious. An isotope brain scan confirmed the clinical suspicion of a left parietal infarct. She died a fortnight later. At necropsy, the heart was considerably enlarged with thrombus formation in the left atrial appendage. Unfortunately no histology was performed. There was evidence of systemic embolisation with multiple infarcts in the kidneys and spleen. The brain showed a large infarct in the distribution of the left middle cerebral artery. Four blocks of spinal cord at different levels were examined (Dr DGF Harriman) and these showed no evidence of anterior horn cell loss. No information on nerve and muscle histology was obtained.

Case 2 SD (1112). A boy born in 1960, was first seen at the age of 13 years. He too, had normal birth and developmental milestones, but was noticed to walk clumsily at the age of three. The disability slowly progressed. The pattern of muscular wasting and weakness was similar to his sister (case 1). Forward flexion of neck was restricted and there was bilateral flexion deformity of the elbows of $90^{\circ}$. Moderate proximal weakness of shoulder girdle muscles was noticed but the biceps and triceps were particularly weak and wasted. Distal muscles of forearms and hands were initially spared. Marked wasting was noticed in the anterior tibials and gastroc- 
nemii and the proximal pelvic girdle muscles were moderately weak. There was no pseudohypertrophy, but total tendon areflexia was noticed. There was no sensory loss. He had fixed equinus deformities of both feet with contractures of tendo Achilles. His colour vision was normal. Serum CPK was 146 units/l (normal 0-50). The muscular weakness gradually progressed with increasing difficulty in walking. In December 1979 (aged 19 years) he complained of breathlessness, epigastric pain and ankle swelling. Within the next six months, he became orthopnoeic with gross congestive failure and jaundice. The muscle weakness had increased considerably but wasting was masked by gross oedema. A chest radiograph showed gross cardiomegaly with pulmonary venous congestion and ECG showed atrial fibrillation, left bundle branch block pattern and unifocal ventricular extrasystoles. An echocardiogram showed dilatation of the left ventricle with an ejection fraction of only $33 \%$. His condition improved with digitalis and diurectis. He was able to sit out of bed but could only walk a few steps. By March 1981, he had become confined to a wheelchair but surprisingly was still able to drive a car. He was not breathless at rest but oedema and hepatomegaly persisted and he was taking a very large dose of diuretics. He recently died at home from cardiac failure at the age of $21 \mathrm{yr}$.

Case 3 CD (1113) was born in 1963 and was the youngest of the three children. She was noticed to have an awkward gait at the age of two years and weakness in the legs and later in the arms was slowly progressive. The pattern of muscle weakness and wasting was similar to the previous cases and she had restriction of neck flexion, bilateral flexion contractures of the elbows to $90^{\circ}$, genu valgum and marked varus deformity of both feet. There was total tendon areflexia but no sensory changes. Her colour vision was normal. Serum CPK (1973) was 145 units/l (normal 0-50), and several ECGs over the years have been normal. Muscle biopsy (Dr DGF Harriman) was reported as follows: "The vastus internus muscle shows much fibrosis and adiposity. Many of the surviving muscle cells are small and have a spotted or lobulated appearance with NADH. But other myofibres are of variable calibre, some particularly, with evidence of fibre splitting and internal nuclei. Both main fibre types are affected. The motor innervation is remarkably preserved and looks abundant because of tissue atrophy; there is no collateral re-innervation, but distal sprouting occurs and many endplates are deformed. Some are reduced to solitary masses. Similar changes are noted in the forearm muscle (flexor carpi ulnaris) but less advanced. There are again many small spotted fibres, and in addition there is a great difference in the degree of affection from fascicle to fascicle. Some resemble the pattern in the vastus internus, others consist mainly of large fibres of the same type (type grouping)." Dr Harriman concluded "the preservation of the innervation places this disorder firmly among the myopathies, although superadded neuropathy, possibly due to nerve compression, may affect the ulnar innervated muscle". Between 1969 and 1980, she had a number of orthopaedic procedures to correct the genu valgum and varus deform- ity of the feet. Following triple arthodesis of both feet, when seen recently (February, 1981), she seemed to be happy that her walking has improved and she could walk 200 yards $(180 \mathrm{~m})$ without help. However, she had great difficulty in climbing stairs. There was moderate weakness of scapular and shoulder gircle muscles with striking wasting in the biceps and triceps. Distal forearm and hand muscles still remained relatively spared. The anterior tibials and gastrocnemii were very thin with moderate weakness and there was wasting of proximal pelvic girdle muscles especially the glutei and quadriceps. Total tendon areflexia and the flexion deformities persisted. There was no facial weakness. She had no cardiac problem.

Case 4 SD (11 7), born in 1929, was the seventh of ten brothers and sisters none of whom was known to have any neuromuscular symptoms. His father was an orphan who died of pulmonary tuberculosis but "always wore surgical boots and had an awkward gait". There has been no history of any similar illness in his mother's family. All three of his children were affected (fig 1). He was first seen in 1976 (age $47 \mathrm{yr}$ ) on account of a right frozen shoulder but was otherwise asymptomatic. Later, he admitted to difficulty in running since the age of 45 years. Examination showed wasting and mild weakness of scapular muscles, biceps and triceps with normal distal arm musculature. Quadriceps and hamstrings were thino and the anterior and posterior tibial muscles wer $\vec{E} \vec{A}$ markedly wasted in a symmetrical fashion. There were n. $\vec{\Phi}$ contractures but he had total tendon areflexia. There waํ no evidence of cardiac failure. Serum CPK waß 112 units/l (normal 0-50). Electromyography of deltoid anterior tibial and quadriceps showed a reduced inter ference pattern with mean duration of potentials frono 8-10 ms and amplitude 1-6 mV. Frequent short duration. and polyphasic potentials were seen, especially in the quadriceps. The heart shadow was enlarged on the chest radiograph and ECG showed complete heart block with a ventricular rate of $36 / \mathrm{min}$ (fig 3). Echocardiography (Dr Dyet) showed slight thickening of the interventricular septum and some anterior movement of the mitral valve in ventricular systole (fig 4). The appearance suggested a hypertrophic cardiomyopathy without obstruction. Left ventricular function was reduced with an ejection fraction of $45 \%$ and a cardiac output of only $1.21 / \mathrm{min}$. Over the last few years, there has been slight deterioration in his muscle power. He has become short of breath on walking half a mile but has had no syncopal attack or angina. He had to retire early but he remains independent and has not required cardiac pacing yet.

\section{Discussion}

MYOPATHY OR NEUROPATHY?

All classes studied in the present family (case 4 and all his three children) had symmetrical muscular weakness and wasting in scapulohumeral distribution in the upper limbs and mostly in tibioperoneal distribution in the lower. Therefore, the cases are described under the general heading of 


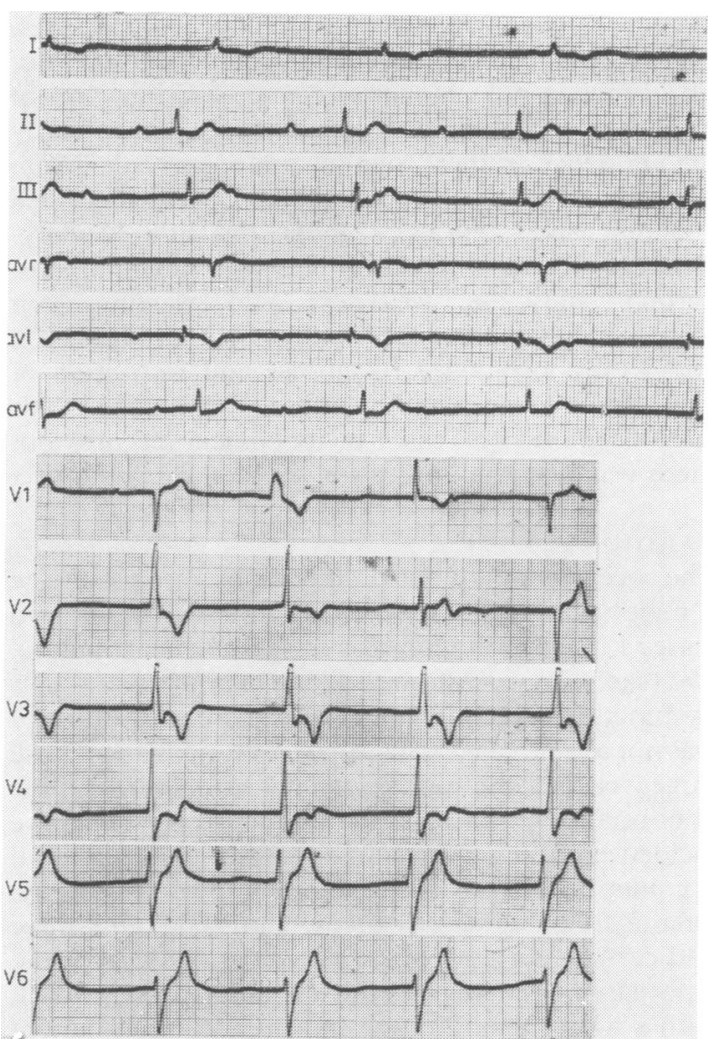

Fig 3 ECG in Case 4 showing complete heart block (ventricular rate 36/min), nodal and ventricular extrasystoles.

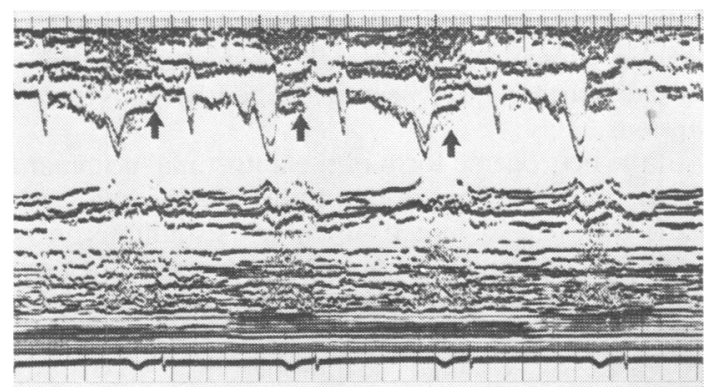

Fig 4 Echocardiogram in Case 4 showing thickening of interventricular septum. The arrows point to the anterior movement of the mitral valve in systole.

scapuloperoneal syndrome. The myopathic basis of the muscular wasting is suggested by the clinical, enzyme and EMG changes and has been demonstrated in the necropsy study of case 1 and in the muscle histology from case 3 . In the former, several blocks of spinal cord were examined and did not show any evidence of anterior horn cell loss, thus excluding any form of spinal muscular atrophy. In the latter, biopsy of the vastus internus showed unequivocal myopathic changes with variation in fibre diameter, internal nuclei, fibre splitting and most important of all preservation of motor innervation and absence of any collateral re-innervation (as seen in chronic neurogenic lesions). "Lobulated" or "spotted" appearance of some fibres with NADH (as seen in this case) have been described in facioscapulo-humeral dystrophy ${ }^{20}$ and also in a patient with malignant hyperpyrexia myopathy. ${ }^{21}$ "These are relatively small type-1 fibres in which thick strands of reaction product are deposited in the peripheral part of the fibre and form walls within the fibre dividing it into compartments." 21

The biopsy from the forearm muscle (in case 3 ) showed similar unequivocal myopathic changes. In places there was evidence of "fibre type grouping", indicative of a neuropathic process which could be interpreted as due to a superadded neuropathy of ulnar compression, this being the only site at which these changes were seen. The patient, unfortunately, refused nerve conduction studies. However a similar admixture of mild neurogenic changes with florid myopathic changes have been described in some cases. ${ }^{5}$ Dystrophic changes have been described in affected muscles in the Kugelberg-Welander disease ${ }^{21} 22$ and "myopathic" changes have been seen in chronically denervated muscle. ${ }^{23}$

Electromyographic studies in case 4 have been non-specific but consistent with a myopathy. The problem of basing diagnosis solely on EMG findings in this particular syndrome, is well illustrated by several previous reports. ${ }^{3-5} 10$ Mixed neurogenic and myogenic features were seen in some cases while in others initial myopathic findings changed to those of denervation at a later date.

\section{GENETICS}

In the present family, the father and all his three children have been affected. Possibly, the children's grandfather was also affected and none of the other nine brothers and sisters of case 4 are known to be affected, and there is no history of any neuromuscular problem in the maternal side. There is no consanguinity. It appears therefore that the inheritance is autosomal dominant. It is very unlikely to be autosomal recessive and certainly not x-linked recessive as two of the children affected are girls, none of whom had any features of Turner's syndrome. Furthermore, if the inheritance had been $x$-linked recessive, both the affected children's mother (wife of case 4) and their grandmother would have to be asymptomatic carriers, which is extremely unlikely, and none of the children were 
found to be abnormal when tested formally for deutan colour blindness. For an $\mathrm{x}$-linked dominant inheritance, transmission from an affected father to an affected son is not possible. With autosomal recessive inheritance, case 4 would have to be homozygous and his wife a heterozygote to have affected all the children: this is extremely unlikely in absence of a consanguinous marriage. The penetration of the gene concerned is obviously variable as only one member in the second generation is known to be affected. The expression of the phenotype is also variable, the neuromuscular problem in the children being much more severe and with earlier age of onset than in the father. Such features are well recognised with autosomal dominant transmission. ${ }^{24}$

\section{CLINICAL ASPECTS}

There appears to be a significant difference in the clinical picture and in the course of the disease seen here, from those described in other families of scapuloperoneal dystrophy (myogenic) with autosomal dominant inheritance. For example, in some of the cases described by Thomas et al ${ }^{9}$ the onset was over the age of $40 \mathrm{yr}$ and the course relatively benign. This is in sharp contrast to the three affected children in the present family, all of whom became severely incapacitated in their teens. However the disease in the father (case 4) resembles the adult onset type ${ }^{9}$ to a great extent. Muscle contractures are characteristically absent in the adult onset type and the significant wasting of the humeral muscles compared to the scapular muscles seen in the three children, is not mentioned in relation to the cases described as autosomal dominant scapuloperoneal myopathy. Difference in the incidence and severity of cardiomyopathy will be discussed later.

Perhaps of greater interest is the striking resemblance the disease in the three children in the present family has to the cases described by Thomas et all as $x$-linked scapuloperoneal dystrophy. The inheritance in the present family, however, is obviously not $x$-linked recessive, as discussed earlier. The similarities include early age of onset, pattern of muscular weakness (affection of upper arm muscles often more severe than those of scapular muscles), elbow contracture, restriction of neck flexion and total tendon areflexia. The incidence and severity of cardiac involvement are also similar. A comparable family has been described by Rotthauwe $e t$ al ${ }^{16}$ but again with $\mathrm{x}$-linked inheritance.

Emery and Dreifuss ${ }^{25}$ described a family of muscular dystrophy with $\mathrm{x}$-linked recessive inheritance but with much slower progression than the classical Duchenne form, which shared many of the features seen in the present family and in the cases described by Thomas et al. ${ }^{17}$ These include the early age of onset, occurrence of elbow contractures, absence of pseudohypertrophy and presence of cardiomyopathy. However, the distribution of weakness and wasting in those cases were clearly scapulopelvic rather than scapuloperoneal. More recently, however, Rowland et al ${ }^{19}$ have described a single case (sporadic) under the title "EmeryDreifuss Muscular Dystrophy" with clear humeroperonal muscular weakness and wasting (not scapulopelvic as described by Emery and Dreifuss ${ }^{25}$ ) and with contractures of elbows and restriction of neck flexion who also had a cardiomyopathy. This case resembles our cases in its clinical features but there was no clear indication as to the inheritance.

\section{CARDIOMYOPATHY}

The occurrence of cardiomyopathy seems to be a major feature in three of our four patients. Two (cases 1 and 2) were in gross congestive heart failure, two (cases 2 and 4) showed significant left ventricular dysfunction on echocardiography (echocardiography was not available when case 1 was studied) and all the three cases showed gross electrocardiographic abnormalities. This is in sharp contrast to the incidence of milder cardiac disease in the autosomal dominant form of the scapuloperoneal dystrophy Of the six cases described by Thomas et al, ${ }^{9}$ three

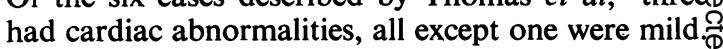
One patient (66 years) had left anterior hemiblock ${ }^{2}-$ with a mitral regurgitation murmur, another patient ${ }_{\mathbb{D}}$ (48 years) showed left axis deviation with deeps Q-waves in leads 11, 111, and AVF; the thire patient (67 years) showed atrial fibrillation witho. antero-lateral wall ischaemia on electrocardiography $\risingdotseq$ Considering the age, some of these changes could be due to coincidental ischaemic heart disease. No cardiac features have been described by Ricker and Mertens. ${ }^{8}$

In the neurogenic form of the autosomal dominant disease no mention of cardiomyopathy has been made. ${ }^{3}$ In the $x$-linked form of scapuloperoneal dystrophy, however, cardiac features are commonly seen. Rotthauwe et al ${ }^{16}$ mentioned nine cases dying suddenly between the ages of 37 and 59 years. Of the six cases of $x$-linked scapuloperoneal dystrophy described by Thomas et al, ${ }^{17}$ cardiomyopathy was a feature of three adult cases (one with atrial fibrillation, one with cardiomegaly and variable P-R interval and another with congestive failure and interventricular conduction disturbance). Two of the patients died. The solitary case described by Rowland $e t a^{19}$ as "Emery-Dreifuss Muscular Dystrophy", which closely resembles our patients, developed permanent "atrial paralysis" and needed pacing. Emery and Dreifuss ${ }^{25}$ described cardiomyopathy in their family with $x$-linked dystrophy 
with scapulopelvic distribution and muscle contracture. The cardiac disorder was also thoroughly analysed by Waters et $\mathrm{al}^{26}$ in two families with an $\mathrm{x}$-linked humeroperoneal syndrome. Emery and Dreifuss ${ }^{25}$ described one case with nodal rhythm, one with atrial fibrillation and another who had "no evidence of atrial activity". Waters et al ${ }^{26}$ described 37 males in two families with an $x$-linked scapuloperoneal syndrome, the neurological features of whom closely resemble our patients and those described by Thomas et al ${ }^{17}$ and Rowland et al. ${ }^{19}$ Muscle histology was equivocal and there is no mention of any changes in the intramuscular nerve fibres. However, the cardiac features were analysed in detail in several patients. Fifteen patients died early, presumably from cardiac cause. Of 15 other patients examined, all showed electrocardiographic abnormalities that ranged from abnormal P-waves to permanent atrial paralysis, and from first degree AV block to complete heart block. Cardiac catheterisation was carried out in five patients, none of whom showed any gross derangement of ventricular function and none of the 15 patients had any clinical evidence of cardiac failure. This is in contrast to our series, where all the three patients with cardiac involvement had either clinical or echocardiographic (or both) evidence of ventricular dysfunction.

In conclusion, the present family illustrates a form of autosomal dominant myopathic scapuloperoneal syndrome with the unusual combination of early age of onset and severe disability by the age of $20 \mathrm{yr}$ in most cases, occurrence of muscle contractures and presence of severe cardiac disease in the majority of the cases. It differs in these aspects from other patients with a similar syndrome with autosomal dominant inheritance, but interestingly resembles the families described with the $\mathrm{x}$-linked form of the disease. Table 2 summarises the important clinical features of the several variants of the scapuloperoneal syndrome (in the light of table 1) and compares them with the features in the present family.

We are grateful to Dr DGF Harriman, Consultant Neuropathologist, Leeds General Infirmary for kindly performing the histological studies in Cases 1 and 3 and to Dr JF Dyet, Consultant Radiologist, Hull Royal Infirmary, for the echocardiographic studies in Cases 2 and 4, and $\operatorname{Dr} C P$ Aber for cardiological advice.

\section{References}

${ }^{1}$ Brossard J. Étude Clinique sur une Forme Héréditaire d'Atrophie Musculaire Progressive débuntant par les Membres Inférieurs (Type Fémoral avec Griffe des Orteils). Paris: Thesis Steinheil, 1886.

${ }^{2}$ Davidenkow S. Scapuloperoneal amyotrophy. Arch Neurol 1939;41:694-701.

${ }^{3}$ Kaeser HE. Scapuloperoneal muscular atrophy. Brain $1965 ; 88: 407-18$.

${ }^{4}$ Mercelis R, Demeester J, Martin J. Neurogenic scapuloperoneal syndrome in childhood. J Neurol Neurosurg Psychiatry 1980;43:888-96.

5 Takahashi K, Nakamura H, Nakashmia R. Scapuloperoneal dystrophy associated with neurogenic changes. J Neurol Sci 1974;23:575-83.

${ }^{6}$ Seitz D. Zur nosologischen Stellung des sogenannten scapuloperonealen syndroms. Disch $Z$ Nervelheilk $1957 ; 175: 547-52$.

' Hausmanowa-Petruseuicz I, Zielinska S. Zur Nozologischen Stellung des scapuloperonealen syndroms. Dtsch Z Nervelheilk 1962;183:377-82.

${ }^{8}$ Ricker K, Mertens HG. The differential diagnosis of the myogenic (Facio)-scapuloperoneal syndrome. Eur Neurol 1968;1:275-307.

${ }^{9}$ Thomas PK, Schott GD, Morgan-Hughes JA. Adult onset scapuloperoneal myopathy. J Neurol Neurosurg Psychiatry 1975;38:1008-15.

${ }^{10}$ Ricker K, Mertens HG, Schimrigh K. The neurogenic scapuloperoneal syndrome. Eur Neurol 1968;1

Table 2 Clinical features of variants of scapuloperoneal syndrome

\begin{tabular}{|c|c|c|c|c|c|}
\hline \multirow{3}{*}{$\begin{array}{l}\text { Features } \\
\text { Pathology } \\
\text { Inheritance }\end{array}$} & \multirow{3}{*}{$\begin{array}{l}\text { Present family } \\
\text { Predominantly } \\
\text { myopathic } \\
\text { Autosomal } \\
\text { dominant }\end{array}$} & \multicolumn{4}{|c|}{ Scapuloperoneal syndrome-other families } \\
\hline & & \multicolumn{2}{|c|}{ Predominantly myopathic } & \multicolumn{2}{|c|}{ Predominantly neurogenic } \\
\hline & & $\begin{array}{l}\text { Autosomal } \\
\text { dominant }\end{array}$ & $\mathrm{X}$-linked recessive & $\begin{array}{l}\text { Autosomal } \\
\text { dominant }\end{array}$ & $\begin{array}{l}\text { Autosomal } \\
\text { recessive }\end{array}$ \\
\hline Age at onset & $\begin{array}{l}\text { Early }<10 \text { yrs } \\
\text { (most cases) }\end{array}$ & Late & Early & Late & Early \\
\hline Course & Rapid (most cases) & Slow & Slow & Slow & Slow \\
\hline Pattern of muscle wasting & $\begin{array}{l}\text { Scapuloperoneal } \\
\text { arm > scap.* }\end{array}$ & Scapuloperoneal & $\begin{array}{l}\text { Scapuloperoneal } \\
\text { arm > scap.* }\end{array}$ & Scapuloperoneal & Scapuloperoneal \\
\hline Early contractures & Present & Absent & Present & Absent & Absent \\
\hline Sensory changes & Absent & Absent & Absent & Present or absent & Present or absent \\
\hline Colour vision & Normal & Normal & Some colourblind & Normal & Normal \\
\hline Cardiomyopathy & Common and severe & Rare and mild & Common and severe & Rare & Rare \\
\hline
\end{tabular}

*Upper arm muscles more wasted than scapular muscles. 
257-74.

${ }^{11}$ Emery ES, Fenichel GM, Eng G. A spinal muscular atrophy with scapuloperoneal distribution. Arch Neurol 1968;18:129-33.

12 Munsat TL. Infantile scapuloperoneal muscular atrophy. Neurology (Minneap) 1968;18:285.

${ }^{13}$ Meadows JC, Marsden CD. Scapuloperoneal amyotrophy. Arch Neurol 1969;20:9-12.

${ }^{14}$ Schwartz MS, Swash M. Scapuloperoneal atrophy with sensory involvement: Davidenkow's syndrome. J Neurol Neurosurg Psychiatry 1975;38:1063-7.

15 Tohgi H, Tsukagoshi H, Toyokura Y. Neurogenic scapuloperoneal syndrome with autosomal recessive inheritance. Clin Neurol (Tokyo) 1971;11:215-20.

${ }^{16}$ Rotthauwe HW, Mortier W, Beyer H. Neuer Typeiner recessiv x-chromosomal vererbten Muskeldystrophie : Scapulo-humero distale Muskeldystrophie mit Frühzeitigen Kontrakturen und Herzrhythmusstörungen. Humangenetik 1972;16:181-200.

17 Thomas PK, Calne DB, Elliott CF. X-linked scapuloperoneal syndrome. J Neurol Neurosurg Psychiatry 1972;35:208-15.

${ }^{18}$ Mawatari S, Katayama K. Scapuloperoneal muscular atrophy with cardiopathy. An x-linked recessive trait. Arch Neurol 1973;28:55-9.

${ }^{19}$ Rowland LP, Fetell M, Olarte M, Hays A, Singh N, Wanat FE. Emery-Dreifuss muscular dystrophy.
Ann Neurol 1979;5:111-7.

${ }^{20}$ Bethlem J, Van Wijngaarden GK, DeJong J. The incidence of lobulated fibres in the facioscapulohumeral type of muscular dystrophy and the limb girdle syndrome. J Neurol Sci 1973;18:351-8.

${ }^{21}$ Harriman DGF. Muscle. In: Blackwood W, Corsellis JAN, ed. Greenfield's Neuropathology. London: Edward Arnold Ltd, 1976:849-902.

22 Pearce J, Harriman DGF. Chronic spinal muscular atrophy. J Neurol Neurosurg Psychiatry 1966;29: 509-20.

${ }^{23}$ Drachman DB, Murphy SR, Nigam MP, Hills JR. "Myopathic" changes in chronically denervated muscle. Arch Neurol 1967;16:14-24.

${ }^{24}$ Emery AEH. Elements of Medical Genetics. Edinburgh and London: Churchill Livingstone, 1975:97-100.

${ }^{25}$ Emery AEH, Dreifuss FE. Unusual type of benign $x$-linked muscular dystrophy. $J$ Neurol Neurosurg Psychiatry 1966;29:338-42.

${ }^{26}$ Waters DD, Nutter DO, Hopkins LC, Dorney ER. Cardiac features of an unusual $x$-linked humeroperoneal neuro-muscular disease. New Engl J Med $1975 ; 293: 1017-22$.

${ }^{27}$ Harding AE, Thomas PK. Distal and scapuloperoneal distribution of muscle involvement occurring within a family with type 1 hereditary motor and sensory neuropathy. J Neurol 1980;224:17-23. 